\title{
Editorial
}

\section{Granzymes in disease: bench to bedside}

\author{
DJ Granville ${ }^{\star, 1}$ \\ Cell Death and Differentiation (2010) 17, 565-566; doi:10.1038/cdd.2009.218
}

Granzymes are a family of structurally related serine proteases that differ markedly in their substrate specificities. Although these proteases were predicted to function as intracellular and/or extracellular proteases in processes ranging from cell death to extracellular matrix cleavage to viral inactivation, ${ }^{1-4}$ intense research over the past two decades has focused primarily on their contributions as intracellular proteolytic mediators of cell death. This can be attributed at least in part to landmark studies by Hayes et al. ${ }^{5}$ demonstrating a synergistic cytotoxic effect when cells were exposed to perforin/cytolysin and granzymes. Since then, and perhaps fostered by the exponential growth of apoptosis research funding in the 1990s, there have been relatively fewer investigations into the potential broader functionality of these proteases with respect to non-apoptotic and/or perforinindependent functions.

There are 5 human granzymes $(\mathrm{A}, \mathrm{B}, \mathrm{H}, \mathrm{K}, \mathrm{M})$ and 11 mouse granzymes ( $A, B, C, D, E, F, G, K, L, M, N)$. One potential explanation for such diversity is to ensure efficient immune-mediated killing of unwanted cells that could otherwise be resistant to the proteolytic activity of a single granzyme. To examine a pan-granzyme role in immunity and disease, perforin knockout mice were, and still are, often used to indirectly determine whether granzymes exert a causative role in disease. If perforin-deficient mice did not elicit a differential response or outcome, it was, and still is, often concluded that the granule pathway is not involved. However, based on emerging evidence, one must question whether using perforin knockout mice exclusively to evaluate a role for granzymes in disease is appropriate. In this issue of Cell Death and Differentiation, a number of articles are provided that summarize the role of granzymes and perforin in a variety of pathologies and present data arguing for both perforin-dependent and perforin-independent functions of these enzymes in cytotoxicity and other responses.

Clearly, one cannot ignore the extensive literature pertaining to the granzyme B/perforin pathway in immune-mediated apoptosis. Indeed, as discussed by Brennan et al., ${ }^{6}$ a complete loss of cytotoxic lymphocyte perforin function results in familial haemophagocytic lymphohistiocytosis, while a partial loss of perforin function appears to strongly predispose patients to haematological malignancies. However, although perforin knockout mice are susceptible to tumorigenesis, the same cannot be said for single granzyme knockout mice, suggesting that it is necessary to prevent the internalization of multiple granzymes and/or other factors to attenuate tumour clearance. Alternatively, it is possible that perforin may possess certain, as yet undefined, granzyme-independent effects on cells.

The granzyme B/perforin pathway has been proposed as the predominant mechanism for immune-mediated apoptosis of allogeneic cells. ${ }^{7}$ As such, this pathway is important in acute and chronic organ transplant rejection. As discussed by Choy, ${ }^{8}$ while findings using perforin or granzyme B knockout mice are mixed for acute rejection due to differences in transplantation models, both molecules appear to be important for T cell-mediated destruction of allogeneic cells and grafts, and both appear to be important in chronic rejection as their absence results in reduced allograft vasculopathy in mouse models of heart transplant rejection. As with most ailments, a role for other granzymes in acute or chronic rejection remains unknown.

Another interesting, potentially perforin-dependent role for granzymes is emerging in diabetes research. As discussed by Thomas et al. ${ }^{9}$ in this issue, perforin deficiency attenuates type I diabetes by reducing islet/beta cell death, suggesting that this pathway is important, while recent evidence presents a role for granzyme-mediated inflammation in this disease. As further support for the granule pathway in disease, mutations in perforin are associated with type I diabetes. As a redundant role for other pro-apoptotic inducers such as FasL has been proposed, it will be important to determine if and how autoantigen production and/or pro-inflammatory cytokine production contribute to diabetes.

An area that remains poorly understood is the role of endogenous granzyme inhibitors in the physiological regulation of granzyme activity and in pathogenesis. As discussed in the review by Kaiserman and Bird, ${ }^{10}$ although inhibitors have been identified for some granzymes, surprisingly little is known pertaining to any pathophysiological role for granzyme serine protease inhibitors (serpins) in disease. Much research needs to be done to verify whether they inhibit granzymes in vivo and whether alterations in their regulation, levels, and/or activity are altered in disease. Further, identification and characterization of granzyme serpins have focused primarily on intracellular inhibitors of apoptosis. Owing to

\footnotetext{
${ }^{1}$ Department of Pathology and Laboratory Medicine, Providence Heart + Lung Institute, St. Paul's Hospital, University of British Columbia, Vancouver, BC, Canada ${ }^{*}$ Corresponding author: DJ Granville, Department of Pathology and Laboratory Medicine, Providence Heart + Lung Institute, St. Paul's Hospital, University of British Columbia, Room 166, Burrard Building, 1081 Burrard Street, Vancouver, BC, Canada V6Z 1 Y6.

Tel: +1 604806 9267; Fax: + 1604806 9274; E-mail: David.Granville@ hli.ubc.ca
} 
emerging evidence suggesting a role for granzyme $A$ in the promotion of inflammation, how do serpins affect this process? Do extracellular inhibitors of any of the granzymes exist in humans? Although many questions exist, it is clear that translational research pertaining to serpins and their contributions to regulating granzymes in disease is an area of unexplored opportunities.

Granzymes also exhibit perforin-independent properties that are relevant to health and disease. Although it has been known for years that granzymes accumulate in the extracellular milieu (i.e., blood, cerebral spinal fluid, synovial fluid, bronchoalveolar lavage) in many chronic inflammatory disease settings, the accumulation of active granzymes has been widely considered to be a consequence of inflammation rather than a cause of disease.$^{11}$ Of note, granzyme $B,{ }^{12}$ and possibly other granzymes, may retain their proteolytic activities in these fluids, which has important therapeutic implications. First, it suggests that extracellular means of regulating granzymes in vivo may be limited. Second, if granzymes do retain their activity, what are the consequences? Indeed, in recent years, several groups have proposed a role in extracellular proteolysis in vitro, but until recently, animal studies of such a phenomenon were severely lacking. Likewise, relevant physiological concentrations of extracellular granzymes, or the presence of granzymes $\mathrm{H}, \mathrm{K}$, and $\mathrm{M}$ in general, require further elucidation. Although there are certainly more questions than answers at this time, many of these ideas are beginning to be addressed. As discussed in-depth in the review by Hendel et al., ${ }^{13}$ emerging studies are beginning to demonstrate a potential perforin-independent, extracellular role for granzymes in disease. ${ }^{14}$ As discussed in the aforementioned review, granzymes are produced by both adaptive and innate immune cells as well as non-immune cells. Further, in certain cases, granzymes are expressed independently of perforin. As such, one can make a strong argument that perforin-dependent effects may not adequately reflect the entire contribution of granzymes in disease.

In another twist, granzyme A, which has traditionally been viewed as a cell death initiator, may not be involved in the induction of cell death at all. Its primary role may be to promote pro-inflammatory cytokine production. ${ }^{15}$ The rationale for this argument is based on observations that high, non-physiological (micromolar) levels of granzyme $A$ and perforin have been historically used to initiate cell death, whereas lower levels of granzyme A induce IL- $\beta$ production from monocytic cells in a perforin-independent manner. Importantly, granzyme A deficiency in these studies attenuated LPS-induced cytotoxicity, suggesting that this protease could have an important death-independent, pro-inflammatory role in infection and sepsis. The implications and emerging roles of granzyme $A$, and possibly granzyme $K$, which is also a tryptase, are reviewed in detail in this issue by Cullen et al. ${ }^{16}$ Indeed, given how difficult it has been over the past two decades to clearly establish a role for granzymes $\mathrm{H}, \mathrm{K}$, and $\mathrm{M}$ in cell death, one has to ponder whether these proteases may also contribute to inflammation and/or other processes. Taken together, a strong argument can now be made for revisiting the conclusions that have been made from translational studies in which perforin knockout mice were used exclusively to assess the role of granzyme-mediated pathways in disease models.

In addition to the induction of cell death and inflammation, alternative pathogenic contributions of granzymes have been proposed. In this issue, Darrah and Rosen ${ }^{17}$ present data supporting a role for granzymes $A, B$, and $H$ in autoantigen production, cytokine secretion, and autoimmunity. However, as with other putative pathogenic roles for granzymes, animal models showing a direct cause-effect relationship between granzymes and autoimmunity are required before reaching any definitive conclusions.

In summary, in addition to immune-mediated cell killing, granzymes may exert other roles in physiology and disease via the production of autoantigens, cleavage of extracellular proteins, regulation of cell migration, platelet function, and in promoting inflammation. Moving forward, it is essential that we start to understand how granzymes are induced in immune/non-immune cells during disease and whether there are mutations that result in enhanced granzyme production and/or activity. Likewise, it is mandatory that we elucidate how these proteases are regulated, confirm the identity of cells that express granzymes in healthy and diseased tissues, assess how their expression or activity is altered in disease, and determine how perforin and serpins are involved in these physiological and pathological processes. Proof-of-concept animal studies in tandem with biochemical and clinical studies are necessary to elucidate pathophysiological processes. The study of granzymes $\mathrm{H}, \mathrm{K}$, and $\mathrm{M}$ with respect to their in vitro activity, let alone their roles in physiology and disease, is still at its infancy and provides great opportunities for discovery. The present review series highlights our current understanding of granzyme biology in the context of both cell death and other proteolytic functions in health and disease. Although many of the reviews raise more questions than answers, it is clear that the research in this exciting area has only just begun.

\section{Conflict of interest}

DJG is a Founder and consultant for viDA Therapeutics, Inc.

1. Kramer MD, Simon MM. Immunol Today 1987; 8: 140-143.

2. Masson D et al. EMBO J 1986; 5: 1595-1600.

3. Masson D, Tschopp J. J Biol Chem 1985; 260: 9069-9072.

4. Masson D, Tschopp J. Cell 1987; 49: 679-685.

5. Hayes MP, Berrebi GA, Henkart PA. J Exp Med 1989; 170: 933-946.

6. Brennan A et al. Cell Death Differ 2010; 17: 607-615.

7. Krupnick AS et al. Transplantation 2002; 74: 871-876.

8. Choy JC et al. Cell Death Differ 2010; 17: 567-576.

9. Thomas HE, Trapani JA, Kay TW. Cell Death Differ 2010; 17: 577-585.

10. Kaiserman D, Bird PI. Cell Death Differ 2010; 17: 586-595.

11. Boivin WA, Cooper DM, Hiebert PR. Lab Invest 2009; 89: 1195-1220.

12. Kurschus FC et al. FEBS Lett 2004; 562: 87-92.

13. Hendel A et al. Cell Death Differ 2010; 17: 596-606.

14. Chamberlain CM et al. Am J Pathol 2010; (in press).

15. Metkar SS et al. Immunity 2008; 29: 720-733.

16. Cullen SP, Brunet M, Martin SJ. Cell Death Differ 2010; 17: 616-623.

17. Darrah E, Rosen A. Cell Death Differ 2010; 17: 624-632. 